\title{
Mycobacterium tuberculosis in the aorta of a patient with takayasu's arteritis. extra pulmonary tuberculosis
}

\author{
María Elena Soto, Virgilia Soto, Julia Isela Martín Sandría, Ricardo Gamboa, Claudia Huesca \\ Immunology and Physiology Departament, Juan Badiano \#1 Colonia Sección XVI, Tlalpan México, D. F, México., \\ *Corresponding Author: virgiliasoto@gmail.com;
}

Received 15 October 2010; revised 2 December 2010; accepted 11 January 2011.

\begin{abstract}
Takayasu's arteritis (TA), of unknown etiology, has been related with Mycobacterium tuberculosis infection. The published cases are controversial. A 29- year-old man died, prolapse of the aortic valve and unspecific arteritis were demonstrated at necropsy. Our objective was to search in the fresh aortic tissues, the infectious agent of tuberculosis through culture, as well as by performing histopathology and searching by the molecular study, sequences of genes associated to mycobacterium and Ziehl's stain. Results. The bacillus of tuberculosis was observed by the Ziehl's stain and the sequences of insertion IS6110 gene were identified. Our findings support the relation of TA with possible tuberculosis infection.Conclusion. The possibility that Takayasu's arteritis is an extra pulmonary tuberculosis must be explored.
\end{abstract}

Keywords: Takayasu's arteritis, extrapulmonary tuberculosis, IS6110 and HupB sequences.

\section{INTRODUCTION}

Takayasu's arteritis (TA) is a chronic inflammatory disease affecting the large arteries and their branches [1]. Pathology is localized in the adventitia, accompanied by mononuclear infiltration and undefined granulomas; the intimae is usually normal but becomes altered in time due to atherosclerosis [2].

TA has been related to Mycobacterium tuberculosis infection, arterial damage depicts granulomatous lesions similar to those of tissues with tuberculosis $[3,4]$ and hyperreactivity to tuberculin application has been documented $[5,6,7,8]$.The published cases are controversial $[9,10,11]$.

We describe an autopsy case, in which unspecific arteritis was found. We searched for tuberculous infection.

\section{DESCRIPTION OF THE CASE}

A 29-year-old man, without previous symptoms of tuberculosis or symptoms relevant to the current illness. No relative with tuberculosis was informed during questioning; no BCG vaccination had been applied to the patient. Three years before, the patient had referred pain in both legs when exerting physically and he had occasional headaches. High blood pressure had been detected in three previous occasions, but he received no antihypertensive treatment.

During indirect questioning, relatives informed that, an hour before his admittance to the hospital, he had complained of an intense oppressive pain in the chest and upper abdomen, pain irradiating to both arms and neck, with difficulty to breath, generalized pulsed headache, and hemoptysis, he seemed disoriented.

Physical exploration revealed a hypertensive crisis, blood pressure of 200/120 mmHg, diaphoresis, we found it confused in time space and person; neck with yugular ingurgitation grade I and murmur in aortic focus the left supraclavicular region, pulseless in the left arm. He presented cardiorespiratory failure half an hour after his admittance, which did not revert with maneuvers of resuscitation. Autopsy was performed by an expert pathologist, finding histopathological data of Takayasu's arteritis.

The case was commented with the rheumatologist, who analyzed the post-mortem clinical file. Demographic data recorded at his arrival at the emergency ward were: age below 40 years, absence of pulse, aortic murmur irradiated to the neck, history of claudication in the lower extremities, these data, provides four clinical criteria, for classification of TA and the autopsy findings which are the gold standard for Takayasu's arteritis diagnosis.

Tissue samples were taken for DNA studies, searching for sequences of the IS6110 gene associated to Mycobacterium tuberculosis. Samples were stains with hematoxylin-eosin, Schiff (periodic acid, PAS), Mason’s trichrome, auramine-rhodamine, and Ziehl-Neelsen.

Ziehl-Neelsen stained and microscopically analyzed. 


\section{MATERIAL AND METHODS}

Autopsy was performed. For the microscopic study, $3-\mu \mathrm{m}$ histological sections of the tissue fixed in $10 \%$ formaldehyde and embedded in paraffin were used. Stains used were, auramine-rhodamine, and Ziehl-Neelsen.

Tissue was embedded in paraffin, and extracted with octane and 10\% alcohol. For paraffin embedding, the Kit Illustra Nucleon BACC Genomic was used. DNA was extracted using a commercial kit (Illustra Nucleón Genomic, GE Healthcare, UK) following manufacturer's instructions. Quantification was performed and readings were made in a spectrophotometer at 260/280 nm.

For DNA amplification of the 123 bp (IS6110) insertion element, the following oligonucleotides were used: IS6110 f (5' CCT GCG AGC GTA GGC GTC GG-3') and IS6110 r (5' CTC GTC CAG CGC CGC TTC GG-3'). Briefly, a $25 \mu \mathrm{L}$ reaction was set up containing $9.3 \mu \mathrm{L}$ of double distilled $\mathrm{H}_{2} \mathrm{O}, 2.5 \mu \mathrm{L}$ of $10 \mathrm{X}$ buffer, $1.5 \mu \mathrm{L}$ of 25 $\mathrm{mM} \mathrm{MgCl} 2,25 \mu \mathrm{M}$ (each) of the four deoxyribonucleoside triphosphate (Epicentre Biotechnologies Company, USA), 2.5U Taq Polymerase (Invitrogen), forward and reverse primer at final concentration of $10 \mathrm{pmol}$ and 2.5 $\mu \mathrm{L}$ of DNA sample (400-500 ng). The following amplification cycle was used for PCR: five min $94^{\circ} \mathrm{C}, 35$ cycles each of 30 seconds at $94^{\circ} \mathrm{C}, 30$ seconds at $60^{\circ} \mathrm{C}$ and $72^{\circ} \mathrm{C}$ for one min, followed by one cycle of $72^{\circ} \mathrm{C}$ for seven min. We used a positive control for the analysis of mycobacterium (M. tuberculosis H37Rv) obtained from cell lines extracted from strains of $M$. tuberculosis HRv37.

\section{RESULTS}

Macroscopic findings were: $1500 \mathrm{ml}$ of blood were found in the left pleural cavity, prolapse of the aortic valve, hypertrophy of the left ventricular wall. The aorta, from its origin until the start of the iliac arteries, presented increased thickness and irregularity of the wall, with some atheroma plaques, in the thoracic aorta, a broken saccular aneurysm was found, measuring $11 \times 8$ $\mathrm{cm}$ in the major axis. Chronic granulomatous inflammation with central necrosis was found in several segments of the aorta (Figure 1). Other regions presented fibrosis with cicatrization alterations in the middle-vascular layer (Figure 2(a)); acid-alcohol resistant bacilli were found in these areas with the Ziehl-Neelsen stain (Figure 2(b)). A blood sample was cultured in search of Mycobacterium tuberculosis, which was positive.

The molecular study identified mycobacterium and the 123 bp that identifies Mycobacterium tuberculosis. Figure 3.

\section{DISCUSSION}

Takayasu's arteritis is generally found in women, it is
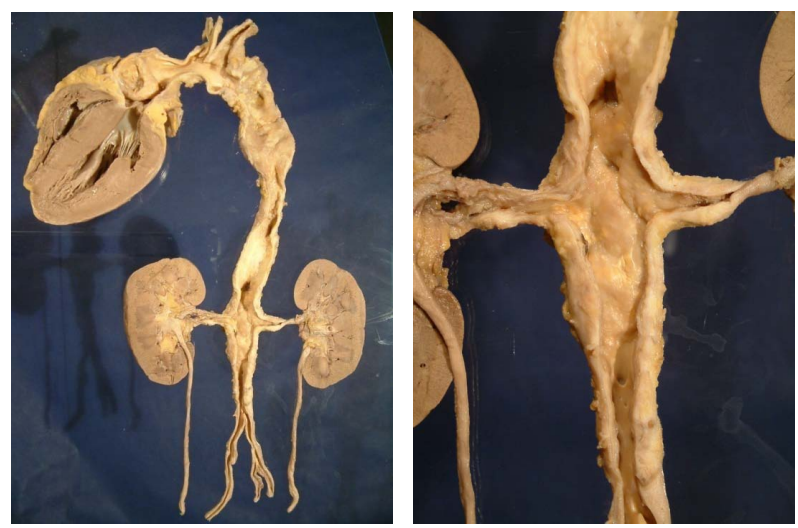

Figure 1. Depict the autopsy specimen constituted by the heart, the aorta, and the kidneys. Prolapse of the aortic valve, thickening and irregularity of the aorta in its diverse segments, with a noticeable constriction of the renal arteries are observed. The right side depicts a close-up of the aorta and of the renal arteries with evident thickening of their walls.

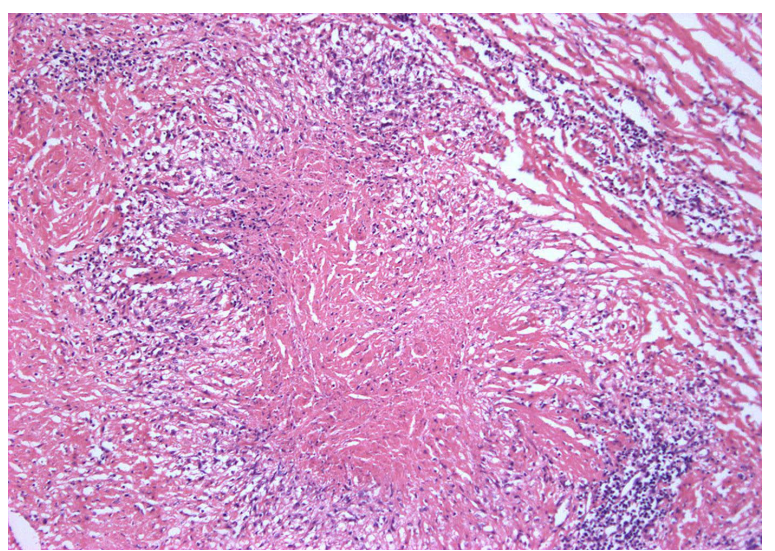

(a)

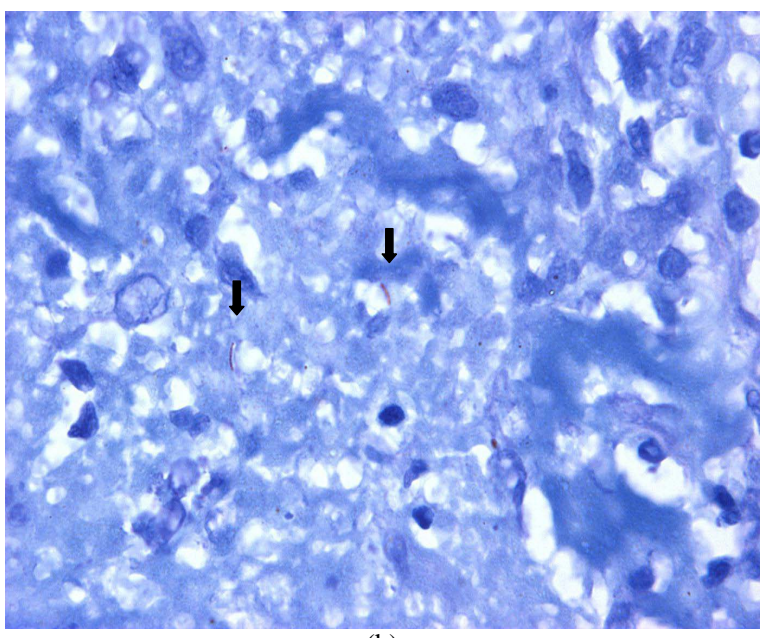

(b)

Figure 2. (a) It cuts to histological stained with hematoxylin and eosin in which granulomatous chronic inflammation with central necrosis in the thickness of the artery is observed aorta. (b) In the black arrow can be seen an acid bacillus resistant alcohol with the stained of Zielh-Neelsen. 


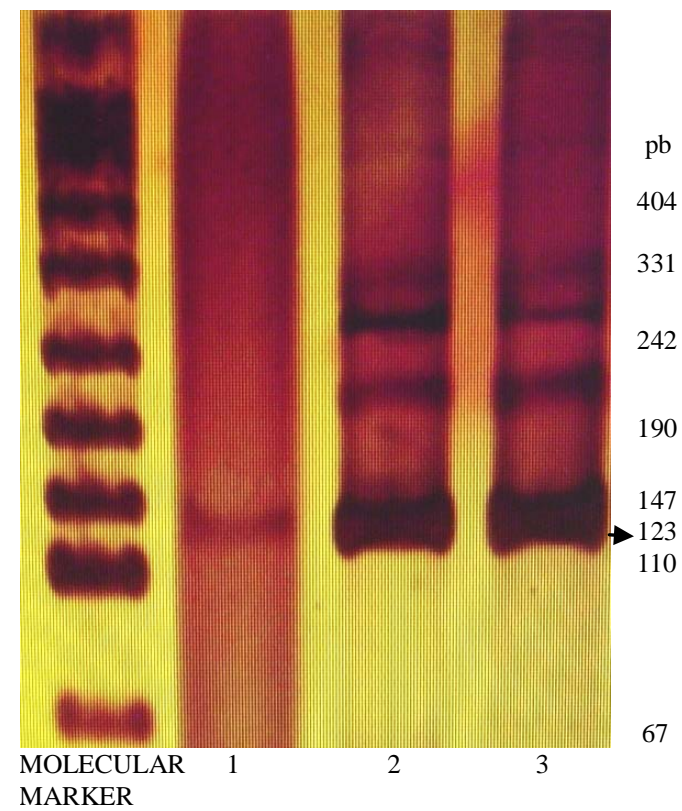

Figure 3. The nitrate silver stained amplification product of $M$. tuberculosis was electrophoresed on nondenaturing $10 \%$ polyacrilamide gel. The 123 product obtained for $M$. tuberculosis is indicated by the arrow. Lanes: molecular size marker; 1 : DNA of bacillus of mycobacterium in the aorta of case; 2 and 3: $M$. tuberculosis DNA H37Rv taken from cellular culture.

rare in men; the relation in Mexico is of 6:1 [7]. Although rare in men, the arterial damage progression is similar. Mycobacterium tuberculosis induces a dominant cellular immune response, similar to that observed in extra- pulmonary tuberculosis. It is feasible that, in TA, arteries are the target organs, which has been demonstrated when the pulmonary arteries are reached by the right blood circulation [12].

For more than five decades, TA has been related with tuberculosis infection, this is still controversial because findings differ; in isolated cases coincidence has been reported [11]. Recently, a prospective study pointed out that there is no evidence for the association with $\mathrm{Myco}$ bacterium tuberculosis in tissues of injured arteries obtained by biopsy [9].

This case presents a man without a previous history of either arteritis or tuberculosis; the reported clinical signs and symptoms were compatible with TA, although no imaging study was performed, the histopathological study was made, and the latter is the gold standard for the diagnosis of TA.

The diagnostic suspicion and its correct classification are necessary as a relevant initial methodology to study the relation of TA with infection.

On the other side, the search for $M$. tuberculosis through culture, specific stains, and modern molecular biology techniques, which have shown diagnostic use- fulness, could provide more reliable results for this association.

\section{CONCLUSION}

This case shows a relation of Takayasu's arteritis with infection by Mycobacterium tuberculosis, and one of the diverse forms of initial clinical manifestation, in the Takayasu's arteritis. These findings must be evaluated by means of systematic studies that would allow the reproducibility of the similar results under the same consensus of search, with molecular techniques used in the taxonomic studies of complex M. tuberculosis.

\section{REFERENCES}

[1] Savory, W. S. (1856) Case of a Young Woman in whom the main Arteries of both Upper Extremities and of the Left Side of the Neck were throughout completely Obliterated. Medico-Chirurgical Transactions, 39, 205-219.

[2] Numano, F. (2000) Vasa vasoritis, vasculitis and atherosclerosis. International Journal of Cardiology, 75, S1 - S8.

[3] Hotchi, M. (1992) Pathological studies on Takayasu arteritis. Heart and Vessels, 7, 11-17.

[4] Pantell, R.H. and Goodman, B.W., Jr. (1981) Takayasu’s arteritis: The relationship with tuberculosis. Pediatrics, 67, 84-88.

[5] Lupi, H. E., Sanchez, T. G. and Castillo, P. U. (1972). Cutaneous reactivity to P.P.D. and antigens of atypical mycobacteria (kansash avium and fortuitum) in patients with unespecific arteritis. Archivos del Instituto de Cardiología de Mexico, 42, 717-725.

[6] Lupi-Herrera, E., Sanchez-Torres, G., Marcushamer, J., Mispireta, J., Horwitz and S. and Vela, J.E. (1977) Takayasu's arteritis. Clinical study of 107 cases. American. Heart Journal, 93, 94-103. doi:10.1016/S0002-8703(77)80178-6

[7] Robles, M. and Reyes, P.A. (1994) Takayasu's arteritis in Mexico: A clinical review of 44 consecutive cases. Clinical and Experimental Rheumatology, 12, 381-388.

[8] Soto, M. E., Espinola, N., Flores-Suarez, L.F.and Reyes, P.A. (2008) Takayasu arteritis: Clinical features in 110 Mexican Mestizo patients and cardiovascular impact on survival and prognosis. Clinical and experimental rheumatology, 26, S9-S15.

[9] Arnaud, L., Cambau, E. and Brocheriou, I. et al. (2009) Absence of Mycobacterium tuberculosis in arterial lesions from patients with Takayasu's arteritis. The Journal of Rheumatology, 36, 1682-1685.

[10] Sagar, S., Ganguly, N.K., Koicha, M.and Sharma, B.K. (1992) Immunopathogenesis of Takayasu arteritis. Heart and Vessels, 7, 85-90. doi:10.1007/BF01744550

[11] Serratrice, J., Benyamine, A. and Vidal, V., et al. (2008) Simultaneous occurrence of diffuse Takayasu's arteritis and severe disseminated tuberculosis. La Revue de Mé decine Interne, 29, 520-523.

[12] Van Vleet, M. E. and Edwards, J. E. (1954) Histologic study of intrapulmonary vessels in tuberculosis. A. M. A. Archives of Pathology, 58, 168-175. 